\title{
Simulation of angular-resolved RABBITT measurements in noble-gas atoms
}

\author{
Alexander W. Bray, Faiza Naseem, and Anatoli S. Kheifets \\ Research School of Physics and Engineering, The Australian National University, Canberra ACT 0200, Australia
}

(Received 15 March 2018; published 8 June 2018)

\begin{abstract}
We simulate angular-resolved RABBITT (reconstruction of attosecond beating by interference of two-photon transitions) measurements on valence shells of noble-gas atoms ( $\mathrm{Ne}, \mathrm{Ar}, \mathrm{Kr}$, and $\mathrm{Xe}$ ). Our nonperturbative numerical simulation is based on solution of the time-dependent Schrödinger equation (TDSE) for a target atom driven by an ionizing XUV and dressing IR fields. From these simulations we extract the angular-dependent magnitude and phase of the RABBITT oscillations and deduce the corresponding angular anisotropy $\beta$ parameter and Wigner time delay $\tau_{W}$ for the single XUV photon absorption that initiates the RABBITT process. Said $\beta$ and $\tau_{W}$ parameters are compared with calculations in the random-phase approximation with exchange (RPAE), which includes intershell correlation. This comparison is used to test various effective potentials employed in the one-electron TDSE. In lighter atoms ( $\mathrm{Ne}$ and $\mathrm{Ar}$ ), several effective potentials are found to provide accurate simulations of RABBITT measurements for a wide range of photon energies up to $100 \mathrm{eV}$ above the valence-shell threshold. In heavier atoms ( $\mathrm{Kr}$ and $\mathrm{Xe}$ ), the onset of strong correlation with the $d$ shell restricts the validity of the single active electron approximation to several tens of $\mathrm{eV}$ above the valence-shell threshold.
\end{abstract}

DOI: 10.1103/PhysRevA.97.063404

\section{INTRODUCTION}

Angular-resolved RABBITT (reconstruction of attosecond beating by interference of two-photon transitions) experiments have been used to coherently control the photoelectron emission direction [1] and, more recently, to measure angulardependent time delay in atomic photoionization [2,3]. These experiments bring sensitive information on ultrafast electron dynamics influenced by correlation and exchange effects. Theoretical modeling of the angular-resolved RABBITT process has been provided within the framework of lowest-order perturbation theory (LOPT) [4,5] and nonperturbatively by solving the time-dependent Schrödinger equation (TDSE) $[2,6]$. In our preceding paper [6], we solved TDSE for a noble-gas atom ( $\mathrm{He}$ or $\mathrm{Ne}$ ) driven by ionizing XUV and dressing IR fields in the configuration of a typical RABBITT measurement. From this solution we deduced the angular dependence of the photoemission time delay as measured by the RABBITT technique [7,8]. Our model was calibrated against a recent angular-resolved measurement on $\mathrm{He}$ [2]. We employed the soft photon approximation (SPA) and used a hydrogenic continuum-continuum (CC) correction to connect the magnitude and phase of the RABBITT oscillations with the angular anisotropy $\beta$ parameter and the Wigner time delay $\tau_{W}$ for the single-XUV-photon absorption that initiates the RABBITT process.

The solution of the TDSE in Ref. [6] was obtained in the single active electron (SAE) approximation and utilized the optimized effective potentials (OEPs) of Sarsa et al. [9]. While such an approach was found to be valid for $\mathrm{He}$, this remains to be shown for $\mathrm{Ne}$ and heavier noble-gas atoms. In the present work, we conduct these tests for noble gases from Ne to Xe by comparing the $\beta$ and $\tau_{W}$ parameters with those coming from calculations performed in the random-phase approximation with exchange (RPAE), the latter including intershell correlation and exchange of the photoelectron with the remaining ionic core. These effects are not included in the TDSE-SAE model. However, the latter model takes an accurate account of ultrafast electron dynamics whereas the RPAE is unable to do so by its basis based construction. In lighter atoms ( $\mathrm{Ne}$ and $\mathrm{Ar}$ ), several effective potentials are found to provide accurate simulation of RABBITT measurements over a wide range of photon energies up to $100 \mathrm{eV}$ above the valence shell threshold. In heavier atoms ( $\mathrm{Kr}$ and $\mathrm{Xe}$ ), the onset of strong correlation with the subvalent $d$ shell restricts the validity of the SAE approximation to several tens of $\mathrm{eV}$ above the valence-shell threshold.

A further goal of the present work is to test the universality of the hydrogenic $\mathrm{CC}$ correction $\left(\tau_{c c}\right)$. This correction relates the single-photon Wigner time delay $\left(\tau_{W}\right)$ and the measured atomic time delay $\left(\tau_{a}\right)$ via

$$
\tau_{a}=\tau_{W}+\tau_{c c}
$$

A hydrogenic CC correction was used in the theoretical analysis of the photoemission time delay measured close to the $3 \mathrm{~s}$ ionization-cross-section minimum in $\mathrm{Ar}$ [10]. The theoretical and experimental time delays reported in Ref. [10] differed by as much as 50 as and no plausible explanation to this disagreement was found to date. We address this issue in the present work. More recently, the RABBITT measurement on $\mathrm{Ne}$ of Isinger et al. [11] has finally reconciled the persistent disagreement between the earlier experiment [12] and a large number of theoretical predictions [13-18]. Our present calculations are similarly in perfect agreement with Ref. [11].

The remainder of this paper is organized as follows: In Sec. II we describe our method and numerical techniques. In Sec. III we present and analyze our numerical data. We conclude by highlighting links with existing experimental measurements and propose several new areas of interest. 


\section{THEORY}

\section{A. Solution of time-dependent Schrödinger equation}

As previously done [6], we solve the one-electron TDSE for a target atom

$$
i \partial \Psi(\boldsymbol{r}) / \partial t=\left[\hat{H}_{\mathrm{atom}}+\hat{H}_{\mathrm{int}}(t)\right] \Psi(\boldsymbol{r}),
$$

where the radial part of the atomic Hamiltonian

$$
\hat{H}_{\text {atom }}(r)=-\frac{1}{2} \frac{d^{2}}{d r^{2}}+\frac{l(l+1)}{2 r^{2}}+V(r)
$$

contains an effective one-electron potential $V(r)$. The various potentials considered are detailed in Sec. II B. The Hamiltonian $\hat{H}_{\text {int }}(t)$ describes interaction with the external field and is written in the velocity gauge

$$
\hat{H}_{\mathrm{int}}(t)=\boldsymbol{A}(t) \cdot \hat{\boldsymbol{p}}, \quad \boldsymbol{A}(t)=-\int_{0}^{t} \boldsymbol{F}\left(t^{\prime}\right) d t^{\prime} .
$$

This external field is comprised of both XUV and IR fields. The XUV field is modelled by an attosecond pulse train (APT) with the vector potential

$$
\begin{aligned}
A_{x}(t)= & \sum_{n=-5}^{5}(-1)^{n} A_{n} \exp \left(-2 \ln 2 \frac{(t-n T / 2)^{2}}{\tau_{x}^{2}}\right) \\
& \times \cos \left[\omega_{x}(t-n T / 2)\right],
\end{aligned}
$$

where

$$
A_{n}=A_{0} \exp \left(-2 \ln 2 \frac{(n T / 2)^{2}}{\tau_{T}^{2}}\right) .
$$

Here, $A_{0}$ is the vector potential peak value and $T=2 \pi / \omega$ is the period of the IR field. The XUV central frequency is $\omega_{x}$ and the time constants $\tau_{x}, \tau_{T}$ are chosen to span a sufficient number of harmonics in the range of photon frequencies of interest for a given atom.

The vector potential of the IR pulse is modelled by the cosine-squared envelope,

$$
A(t)=A_{0} \cos ^{2}\left(\frac{\pi(t-\tau)}{2 \tau_{\mathrm{IR}}}\right) \cos [\omega(t-\tau)] .
$$

The IR pulse is shifted relative to the APT by a variable delay $\tau$ such that the RABBITT signal of the even- $2 q$ sideband (SB) oscillates as

$$
S_{2 q}(\tau)=A+B \cos [2 \omega \tau-C] .
$$

The solution of the TDSE (2) is found by using the iSURF method as given in Morales et al. [19]. A typical calculation with XUV and IR field intensities of $5 \times 10^{9}$ and $3 \times 10^{10} \mathrm{~W} / \mathrm{cm}^{2}$, respectively, would take up to $35 \mathrm{CPU}$ hours for each $\tau$.

The RABBITT parameters $A, B$, and $C$ entering Eq. (7) can be expressed via the absorption and emission amplitudes

$$
\begin{aligned}
& A=\left|\mathcal{M}_{k}^{(-)}\right|^{2}+\left|\mathcal{M}_{k}^{*(+)}\right|^{2}, \quad B=2 \operatorname{Re}\left[\mathcal{M}_{k}^{(-)} \mathcal{M}_{k}^{*(+)}\right], \\
& C=\arg \left[M_{k}^{(-)} M_{k}^{*(+)}\right]=2 \omega \tau_{a} .
\end{aligned}
$$

Here, $\mathcal{M}_{k}^{( \pm)}$are complex amplitudes for the angle-resolved photoelectron produced by adding or subtracting an IR photon, respectively. By adopting the soft photon approximation (SPA) [20] we can write

$$
\begin{aligned}
A, B & \propto\left|J_{1}\left(\boldsymbol{\alpha}_{0} \cdot \boldsymbol{k}\right)\right|^{2}|\langle f|z| i\rangle|^{2} \\
& \propto\left[1+\beta P_{2}\left(\cos \theta_{k}\right)\right] \cos ^{2} \theta_{k} .
\end{aligned}
$$

Here we made a linear approximation to the Bessel function because the parameter $\alpha_{0}=F_{0} / \omega^{2}$ is small in a weak IR field. See the Appendix for a more detailed derivation. In Eq. (9), $\theta_{k}$ is the angle between the photoelectron emission direction $\hat{\boldsymbol{k}}$ and the electric-field vector of the linearly polarized light. By fitting the calculated angular dependence of the $A$ and $B$ parameters with the SFA expression (9) we can obtain the two sets of the angular anisotropy parameters $\beta_{A}^{\mathrm{SB}}$ and $\beta_{B}^{\mathrm{SB}}$ and compare them with the value calculated by the RPAE model. At the same time, we derive the angular dependence from the odd high-harmonic $(\mathrm{HH})$ peaks by fitting angular variation of their amplitude with $1+\beta^{\mathrm{HH}} P_{2}\left(\cos \theta_{k}\right)$. Thus, for each target atom three sets of $\beta$ parameters are extracted and analyzed over a wide photon energy range.

Laurent et al. [1] proposed a different parametrization of the angular dependence of the RABBITT signal. In the case for which the APT has only odd HH peaks, it reads

$$
\begin{aligned}
F_{q}\left(\theta_{k}, \tau\right) & =\sum_{j=0}^{2 L_{\max }} \beta_{j}(q, \tau) P_{j}\left(\cos \theta_{k}\right) \\
& \propto 1+\beta_{2} P_{2}\left(\cos \theta_{k}\right)+\beta_{4} P_{4}\left(\cos \theta_{k}\right) .
\end{aligned}
$$

While $\beta_{2}$ in Eq. (10) is identical with our definition of $\beta^{\mathrm{HH}}, \beta_{2}$ and $\beta_{4}$ can be expressed via $\beta^{\mathrm{SB}}$. By expanding Eq. (9) over the Legendre polynomials, we arrive at the following expressions:

$$
\beta_{2}=\frac{70+55 \beta^{\mathrm{SB}}}{35+14 \beta^{\mathrm{SB}}}, \quad \beta_{4}=\frac{36 \beta^{\mathrm{SB}}}{35+14 \beta^{\mathrm{SB}}} .
$$

In the following, we show that, in all presently studied cases, $\beta^{\mathrm{HH}} \simeq \beta_{A}^{\mathrm{SB}} \simeq \beta_{B}^{\mathrm{SB}}$ and one set of $\beta$ parameters fits all the RABBITT measurements. The $\beta_{4}$ and $\beta_{2}$ parameters depend on this $\beta$ linearly. Thus, the $\beta_{4}$ parameter is redundant and its introduction by Cirelli et al. [3] is superfluous.

The $C$ parameter is converted to the atomic time delay $\tau_{a}$ by Eq. (8) and analyzed as a function of the photoelectron direction relative to the polarization axis. The angular dependence of $\tau_{a}$ is compared with the analogous dependence of the Wigner time delay $\tau_{\mathrm{W}}$ [21]. The time delay difference $\tau_{a}-\tau_{\mathrm{W}}$ in the zero-angle direction is compared with the hydrogenic CC correction $\tau_{\mathrm{CC}}[22]$.

\section{B. One-electron potential}

In our previous work on $\mathrm{He}$ and $\mathrm{Ne}$, we employed an optimized effective potential (OEP) [9]. This potential is derived by a simplified treatment of the exchange term in the Hartree-Fock (HF) equations using the Slater X- $\alpha$ ansatz [23]. The OEP potential takes the form

$$
\begin{aligned}
V_{e}(r) & =-\frac{1}{r}\left(1+\left(Z_{0}-1\right) \sum_{p=0}^{S} \sum_{k=1}^{n_{p}} c_{k, p} r^{p} e^{-\beta_{k, p} r}\right) \\
& \equiv-\frac{Z^{*}(r)}{r}
\end{aligned}
$$



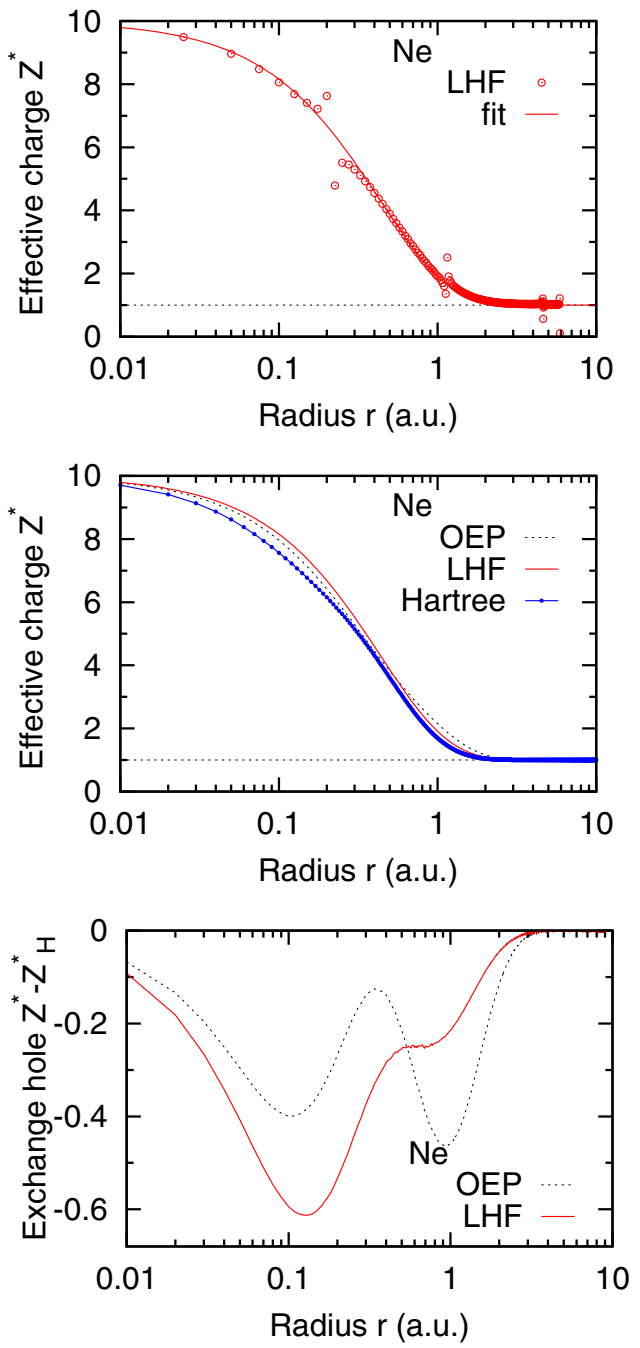

FIG. 1. The effective charge $Z^{*}(r)=-r V(r)$ of Ne generated from various one-electron potentials. (top) The effective charge $Z^{*}$ derived from the LHF potential using the HF radial orbital $\kappa=0.01$ and $\ell=0$ is shown with the (red) open circles. The fit with the analytic expression (15) is shown by the (red) solid line. (middle) The effective charges generated from the optimized effective potential (OEP) of Ref. [9] and the LHF potential are compared with the spherically symmetric Hartree potential (13). (bottom) The charge difference (exchange hole) $Z^{*}-Z_{\mathrm{H}}^{*}$ for the OEP and LHF potentials.

where the effective charge $Z^{*}(r)$ varies from the unscreened nucleus charge $Z_{0}$ as $r \rightarrow 0$ and unity at large distances $r \rightarrow$ $\infty$. The former limit is satisfied by imposing the condition $\sum_{k=1}^{n_{0}} c_{k, 0}=1$. The effective charges $Z^{*}(r)$ for $\mathrm{Ne}$ and $\mathrm{Ar}$ are shown in Figs. 1 and 2, respectively.

It is instructive to compare $Z^{*}$ with the effective charge derived from the spherically symmetric part of the Hartree potential $Z_{\mathrm{H}}^{*}=Z_{0}-r V_{\mathrm{H}}(r)$, where

$$
\begin{aligned}
V_{\mathrm{H}}(r) & =\frac{1}{4 \pi} \int d \Omega_{r} \int_{0}^{\infty} d \boldsymbol{r}^{\prime} \frac{\rho\left(\boldsymbol{r}^{\prime}\right)}{\left|\boldsymbol{r}^{\prime}-\boldsymbol{r}\right|}, \\
\rho\left(\boldsymbol{r}^{\prime}\right) & =\sum_{n l m}\left|\psi_{n l m}\left(\boldsymbol{r}^{\prime}\right)\right|^{2} .
\end{aligned}
$$
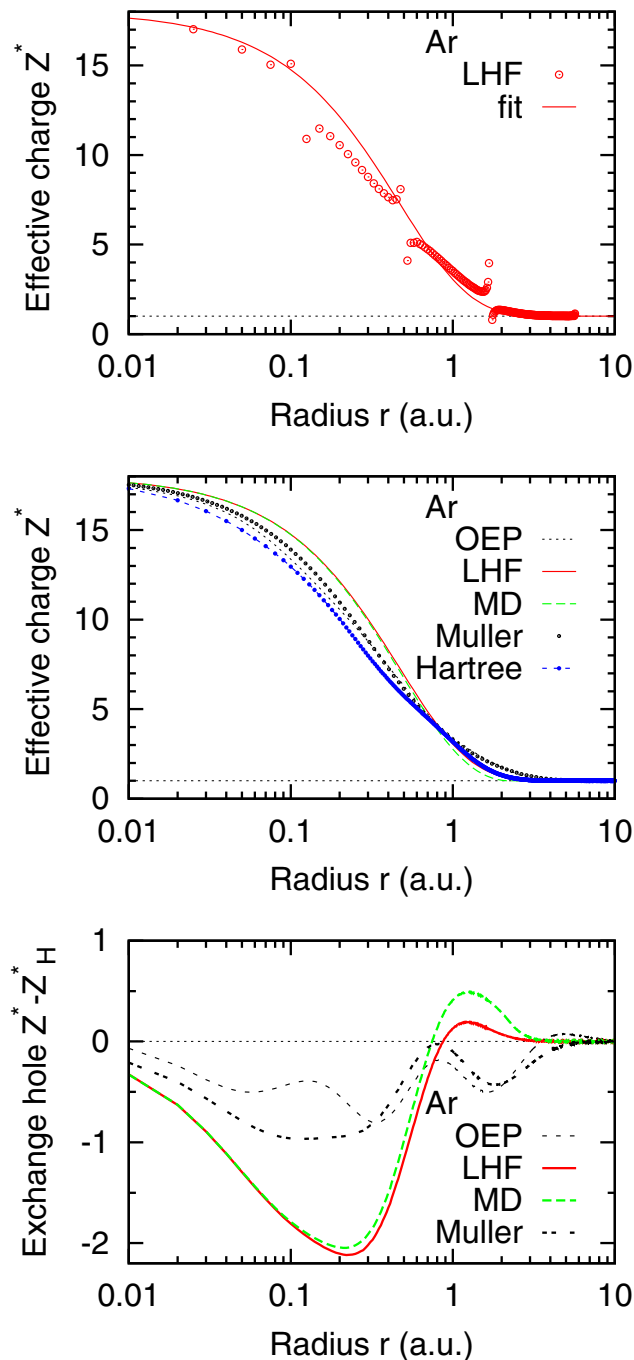

FIG. 2. Same as Fig. 1 for argon. In addition, the effective charge $Z^{*}(r)=-r V(r)$ is generated from the atomic pseudopotential of Miller and Dow [24] (labeled MD) and the Muller potential [7].

By way of spherical integration, the above expression can be reduced to the following radial integral:

$$
V_{\mathrm{H}}(r)=\int_{0}^{\infty} r^{\prime 2} d r^{\prime} \frac{\rho\left(r^{\prime}\right)}{r_{>}}, \quad \rho\left(r^{\prime}\right)=\sum_{n l}^{N-1}\left|R_{n l}\left(r^{\prime}\right)\right|^{2} .
$$

Here, $r_{>}=\max \left(r, r^{\prime}\right)$ and the upper limit in the sum $N-1$ indicates that the number of electrons in the singly ionized atomic core is reduced by one. The charge $Z_{\mathrm{H}}^{*}$ is derived from the charge density of the occupied atomic orbitals and it neglects the exchange of the departing photoelectron with those in the core. Thus, $Z_{\mathrm{H}}^{*}$ provides a convenient baseline for elucidating the exchange effects. The charge difference $Z^{*}-Z_{\mathrm{H}}^{*}$ is expected to be negative as the exchange softens the atomic core and reduces its screening capacity. In density functional theory (DFT), this effect is termed the exchange and correlation hole [25].

A further model potential that we employ is that of a localized Hartree-Fock (LHF) potential generated from a known continuous orbital calculated in a frozen HF core [26]. 
TABLE I. The valence-shell energies, in atomic units, calculated with various model potentials. The experimental thresholds are from Ref. [27]. The LHF entries also contain the $\alpha$ parameters from Eq. (15).

\begin{tabular}{lcccc}
\hline \hline Method & $\mathrm{Ne} 2 p$ & $\operatorname{Ar} 3 p$ & $\mathrm{Kr} 4 p$ & $\mathrm{Xe} \mathrm{5p}$ \\
\hline Expt. [27] & 0.792 & 0.579 & 0.514 & 0.445 \\
HF & 0.850 & 0.591 & 0.524 & 0.457 \\
OEP [9] & 0.851 & 0.590 & 0.528 & 0.467 \\
LHF & $0.843(2.29)$ & $0.583(2.11)$ & $0.202(2.80)$ & $0.412(2.54)$ \\
Muller [7] & & 0.581 & & \\
MD [24] & & 0.423 & 0.203 & \\
\hline \hline
\end{tabular}

The radial Schrödinger equation with the atomic Hamiltonian (3) can be rewritten such that the LHF is expressed in terms of the known HF radial orbital and its second derivative,

$$
V_{\mathrm{HF}}(r)=\frac{\kappa^{2}}{2}-\frac{\ell(\ell+1)}{2 r^{2}}+\frac{P_{\kappa \ell}^{\prime \prime}(r)}{P_{\kappa \ell}(r)} .
$$

The LHF should be weakly sensitive to the choice of the momentum $\kappa$ and the orbital momentum $\ell$. For practical reasons, we chose $\kappa=0.01$ and $\ell=0$ to avoid multiple nodes of $P_{\kappa \ell}(r)$ where the right-hand side of Eq. (14) diverges. The effective charge $Z^{*}=-r V_{\mathrm{HF}}(r)$ derived from Eq. (14) is a smooth function outside of these nodes and can be fit with an analytical expression

$$
Z_{\mathrm{HF}}^{*}(r)=\left(Z_{0}-1\right) e^{-a r}+1
$$

This fit with $a=2.29$ for Ne and $a=2.11$ for Ar is shown in the top panels of Figs. 1 and 2, respectively.

The $p=0$ term in Eq. (12) is analogous to the Muller potential introduced specifically for Ar [7],

$$
V_{\mathrm{M}}(r)=-\frac{1}{r}[1+5.4 \exp (-r)+11.6 \exp (-3.682 r)] .
$$

Miller and Dow [24] suggested an alternative analytical expression

$$
Z_{\mathrm{MD}}^{*}(r)=1+\frac{\left(Z_{0}-1\right)(1-r / R)^{2} \theta(R-r)}{1+C r+D r^{2}},
$$

where $\theta(R-r)$ is the unit step function. The numerical parameters $R, C$, and $D$ are chosen to match the variation of the angular anisotropy parameter $\beta$ with energy across the Cooper minimum (CM) known from experiment. The effective charges generated with the potentials (16) and (17) for Ar are shown in Fig. 2 along with those extracted from the OEP and LHF potentials. As compared with $\mathrm{Ne}$, the role of exchange is significantly larger in Ar with the corresponding exchange hole being much greater. We also note that charge difference $Z^{*}-Z_{\mathrm{H}}^{*}$ in argon with the LHF, and particularly $\mathrm{MD}$, potentials is slightly positive at larger distances.

The valence-shell energies calculated with various model potentials along with the experimental threshold energies are compiled in Table I. For the LHF potential, we also show in parentheses the $\alpha$ parameters from Eq. (15).
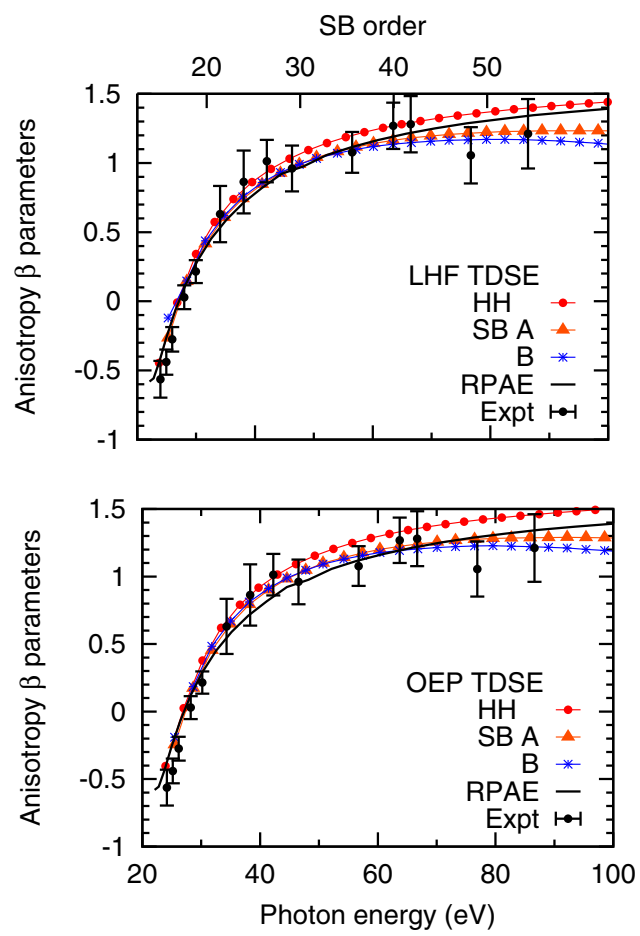

FIG. 3. Angular anisotropy $\beta$ parameters for the Ne $2 p$ valence shell extracted from the TDSE calculations with the LHF potential (top) and the OEP potential (bottom). The $\beta^{\mathrm{HH}}$ parameters extracted from the angular dependence of the high-harmonic peaks are plotted with (red) filled circles. Same parameters $\beta^{\mathrm{SB}}$ extracted from the angular variation of the RABBITT $A$ and $B$ coefficients in Eq. (8) are plotted with (orange) triangles and (blue) asterisks, respectively. The RPAE calculation is shown by the solid line. The experiment [28] is given by the points with the error bars.

\section{RESULTS}

\section{A. Neon $2 p$ shell}

In Fig. 3 we display the angular anisotropy $\beta$ parameters for the Ne $2 p$ valence shell extracted from the TDSE calculations with the LHF potential (top) and the OEP potential (bottom). The $\beta^{\mathrm{HH}}$ parameters extracted from the angular dependence of the high-harmonic peaks are plotted along with the $\beta^{\mathrm{SB}}$ parameters extracted from the angular variation of the RABBITT $A$ and $B$ parameters in Eq. (8). The RPAE calculation is shown with the solid line. This calculation is known to reproduce accurately the experimental $\beta$ parameters across the studied photon energy range [28].

We see that the harmonics and sideband TDSE calculations of $\beta$ parameters are consistent between each other and are fairly close to the XUV-only RPAE calculation, with the LHF results marginally closer to the RPAE than the OEP ones. In our previous work [6] we employed the OEP potential and quoted $\beta^{\mathrm{SB}} \simeq 0.3$ for sideband 20 (SB20) which is in reasonable agreement with the present results for both potentials.

The angular dependence of the atomic time delay $\tau_{a}\left(\theta_{k}\right)$ as a function of the escape angle is shown in Fig. 4. The top and middle panels display the TDSE calculations with the LHF and OEP potentials, respectively. The bottom panel shows the angular dependence of the Wigner time delay $\tau_{\mathrm{W}}\left(\theta_{k}\right)$ from the XUV-only RPAE calculation. We see that both TDSE 

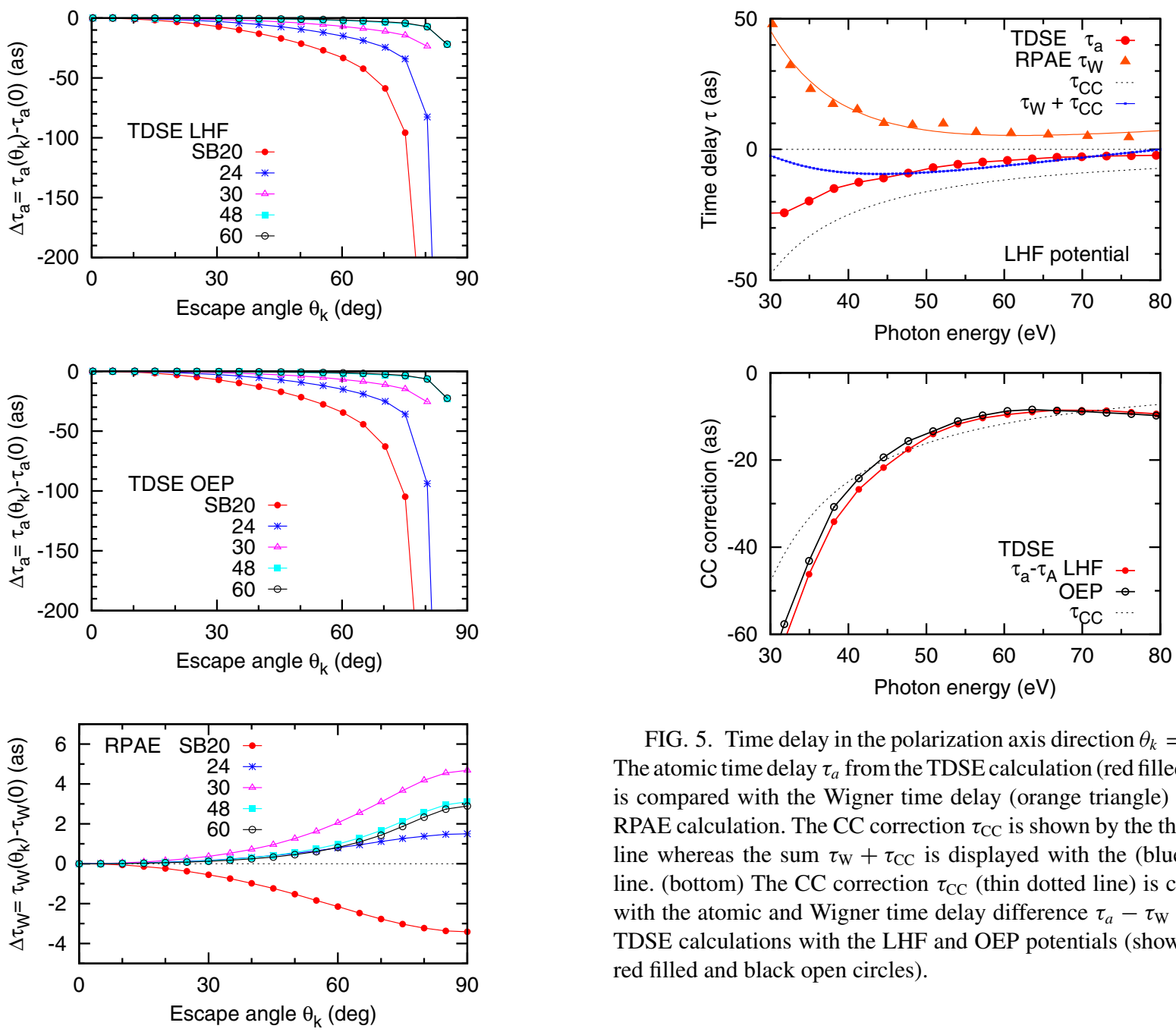

FIG. 5. Time delay in the polarization axis direction $\theta_{k}=0$. (top) The atomic time delay $\tau_{a}$ from the TDSE calculation (red filled circles) is compared with the Wigner time delay (orange triangle) from the RPAE calculation. The CC correction $\tau_{\mathrm{CC}}$ is shown by the thin dotted line whereas the sum $\tau_{\mathrm{W}}+\tau_{\mathrm{CC}}$ is displayed with the (blue) dotted line. (bottom) The CC correction $\tau_{\mathrm{CC}}$ (thin dotted line) is compared with the atomic and Wigner time delay difference $\tau_{a}-\tau_{\mathrm{W}}$ from the TDSE calculations with the LHF and OEP potentials (shown by the red filled and black open circles).

FIG. 4. Angular variation of the atomic time delay $\Delta \tau_{a}=$ $\tau_{a}\left(\theta_{k}\right)-\tau_{a}(0)$ in various sidebands of the Ne $2 p$ RABBITT trace calculated with the LHF potential (top) and the OEP potential (middle). (bottom) Angular variation of the Wigner time delay $\Delta \tau_{\mathrm{W}}=$ $\tau_{\mathrm{W}}\left(\theta_{k}\right)-\tau_{\mathrm{W}}(0)$ from the XUV-only RPAE calculation.

calculations are quite close to one another while the RPAE calculation suggests an angular dependence which is an order of magnitude weaker. The consequence being that nearly all the angular dependence of the atomic time delay in $\mathrm{Ne}$ comes from the $\mathrm{CC}$ correction introduced by the probe IR field. A similar observation was made for He where the Wigner time delay is isotropic [2]. In Ne, the Wigner time delay is not entirely isotropic because the $2 p \rightarrow \epsilon s$ and $2 p \rightarrow \epsilon d$ channels enter the ionization amplitude with their own spherical harmonics; namely, $Y_{00}\left(\theta_{k}\right)$ and $Y_{20}\left(\theta_{k}\right)$. However, as a result of the Fano propensity rule [29], the $d$ continuum is strongly dominant and the $s$ continuum contributes only a very weak angular modulation. We note that this situation would change drastically near the CM in $\mathrm{Ar}$ and heavier noble gases where the angular dependence of the Wigner time delay is very strong.

The time delay in the polarization axis direction $\theta_{k}=0$ is shown in Fig. 5. In the top panel, we compare the atomic time delay from the TDSE calculation with the LHF potential

and the Wigner time delay $\tau_{\mathrm{W}}$ from the RPAE calculation. The hydrogenic CC correction $\tau_{\mathrm{CC}}$, which is shown separately, is then added to the Wigner time delay. This correction, as a function of the photoelectron energy, is represented by the analytic expression

$$
\tau_{\mathrm{CC}}(E)=N E^{-3 / 2}[a \ln (E)+b],
$$

where the coefficients $N, a$, and $b$ are found from fitting the regularized continuum-continuum delay shown in Fig. 7 of Ref. [22]. We see that, except for the near-threshold region where the photoelectron energy is very small and where the regularization of $\tau_{\mathrm{CC}}$ may not be applicable, the identity (1) $\tau_{a} \simeq \tau_{W}+\tau_{c c}$ holds very well.

This utility of the hydrogenic CC correction can be used to analyze the recent set of RABBITT measurements on $\mathrm{Ne}$ [11] where the time-delay difference between the $2 s$ and $2 p$ shells in Ne was determined. This analysis is shown in Fig. 6. In the top panel, we plot the Wigner time delay from the RPAE calculation for the individual $2 s$ and $2 p$ shells and their difference. In the bottom panel, the Wigner time-delay difference is augmented by that of the CC correction. We assume that the $\mathrm{CC}$ correction $\tau_{\mathrm{CC}}$ is a universal function of the photoelectron energy and as such the CC correction difference between shells at the same photon energy is caused by their 

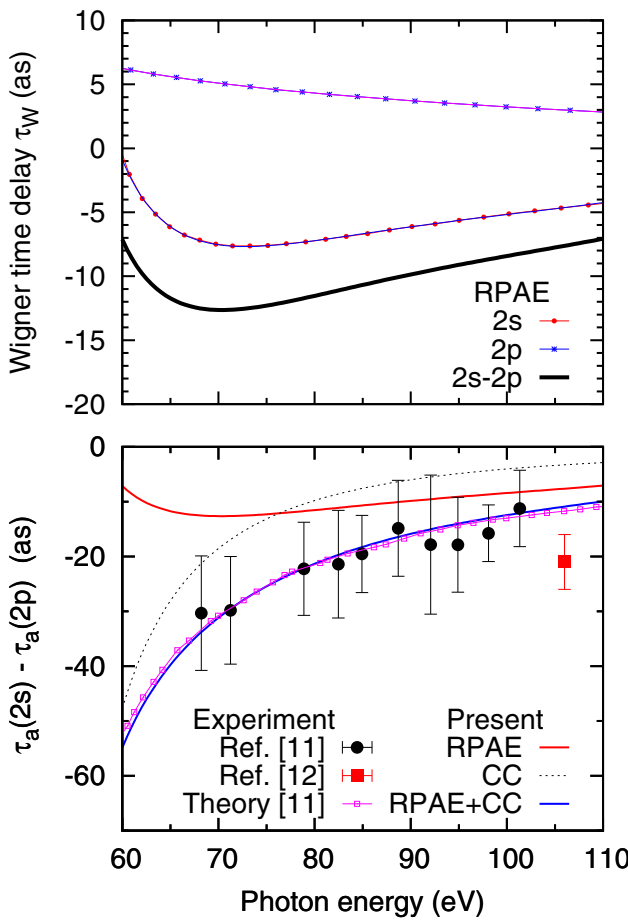

FIG. 6. (top) Wigner time delay in the $2 s$ and $2 p$ shells of $\mathrm{Ne}$ and their difference. (bottom) Atomic time-delay difference $\tau_{a}(2 s)-$ $\tau_{a}(2 p)$ as measured experimentally by Isinger et al. [11] (filled circles) and Schultze et al. [12] (red square). The Wigner time delay difference $\tau_{\mathrm{W}}(2 s)-\tau_{\mathrm{W}}(2 p)$ (red solid line) is augmented by the CC correction difference $\tau_{\mathrm{CC}}(2 s)-\tau_{\mathrm{CC}}(2 p)$ (dotted line) to get the atomic time delay difference $\tau_{a}(2 s)-\tau_{a}(2 p)$ (blue solid line) which is compared with the calculated result of Isinger et al. [11] (purple squares).

varying ionization potentials. The atomic time delay difference

$$
\tau_{a}(2 s)-\tau_{a}(2 p)=\tau_{\mathrm{W}}(2 s)-\tau_{\mathrm{W}}(2 p)+\tau_{\mathrm{CC}}(2 s)-\tau_{\mathrm{CC}}(2 p)
$$

is compared with the RABBITT measurement and the RPA calculation presented in Ref. [11]. We see that both calculations (almost indistinguishable in the scale of the figure) reproduce the measurement [11] very well. In contrast, the older measurement [12] deviates from the theoretical predictions by nearly a factor of two.

\section{B. Argon $3 p$ shell}

The $\beta$ parameters for the $\operatorname{Ar} 3 p$ shell extracted from the angular dependence of the high-harmonic peaks and sidebands are shown in Fig. 7. The TDSE calculations performed with the LHF and OEP potentials are shown in the top and bottom panels, respectively. The three sets of $\beta$ parameters are compared with the RPAE calculation and with experiment [30]. We observe from this figure that all three sets of $\beta$ parameters extracted from the TDSE calculation with the LHF potential follow closely the RPAE prediction and agree with experiment. At the same time, the OEP TDSE results are displaced relative to the RPAE in the photon energy scale by as much as $10 \mathrm{eV}$. This mismatch is a reflection of the displacement of the CM position in the photoionization cross section. This position can
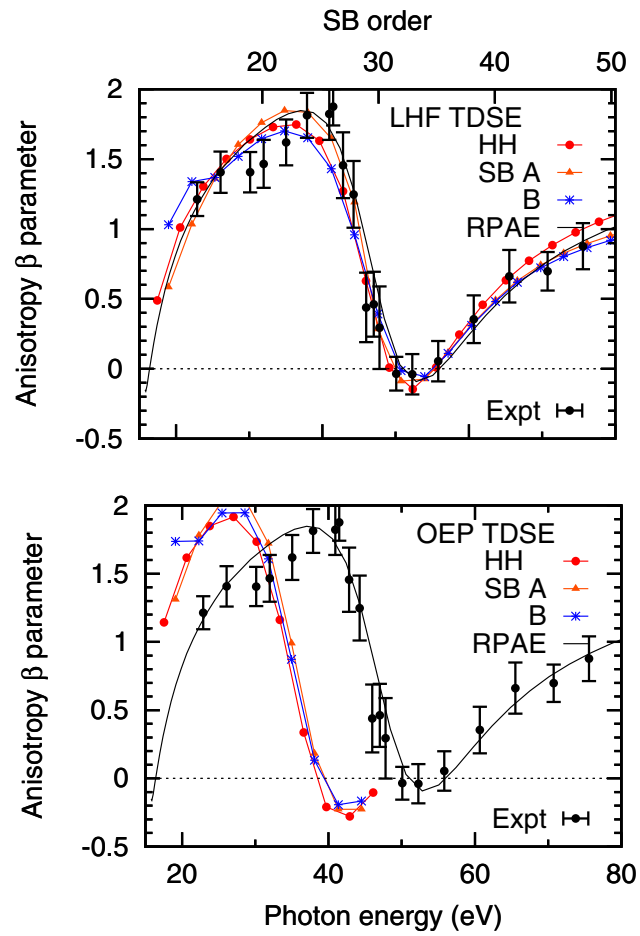

FIG. 7. Same as Fig. 3 for Ar $3 p$ shell. The experiment [30] is given by the points with error bars.

be located very accurately from the squared radial integral [31]

$$
\left|\int_{0}^{\infty} P_{3 p}(r) P_{E d}(r) r d r\right|^{2}
$$

A plot of this integral is given in Fig. 8 where the radial orbitals of the bound and continuous states have been calculated from the Schrödinger equation with Hamiltonian (3) using the LHF, OEP, Muller [7] and Miller and Dow [24] potentials. The equivalent value from the HF and RPAE calculations are also shown. We see that the CM position is misplaced for each of the potentials except the LHF. Subsequently, in the following, we present our TDSE results calculated with the LHF potential only.

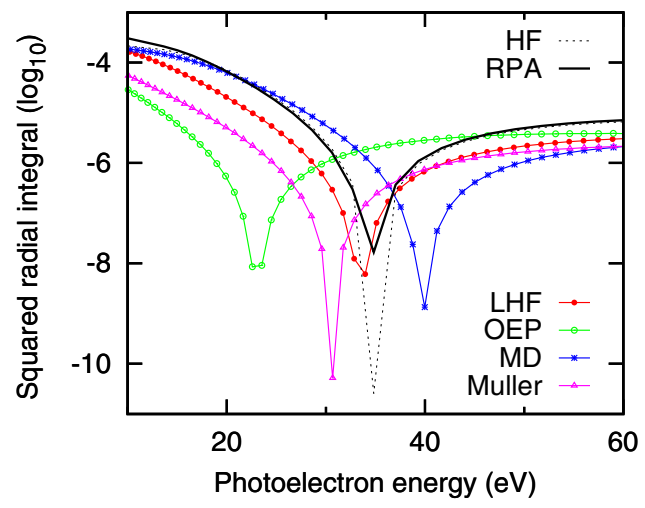

FIG. 8. Squared radial integral (20) calculated with the LHF (red filled circles), OEP (open green circles), Miller and Dow [24] (blue asterisks), and Muller [7] (purple triangles) potentials for Ar. The HF and RPAE results are shown with black dotted and solid lines, respectively. 

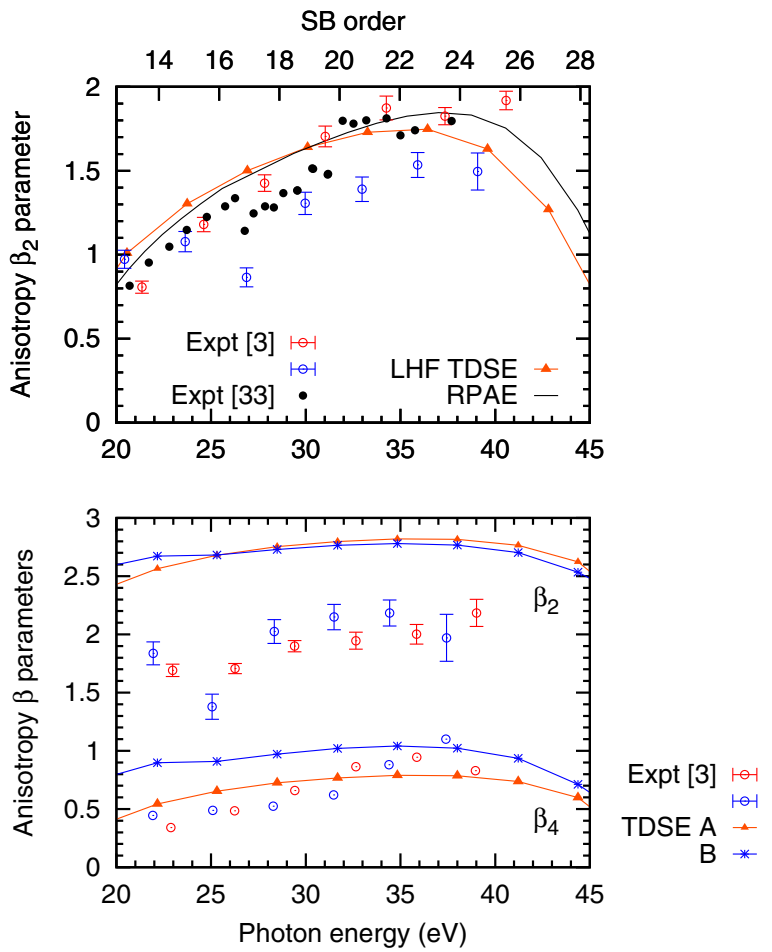

FIG. 9. (top) Anisotropy parameters derived from the angular variation of the high-harmonic peaks. The two sets of experimental values $\beta_{2}$ from Ref. [3] are shown by open circles with error bars. The synchrotron measurement [32] is shown by black dots. The orange triangles connected with a solid line visualize $\beta^{\mathrm{HH}}$ from the LHF TDSE calculation whereas the black solid line displays the RPAE result. (bottom) Experimental $\beta_{2}$ and $\beta_{4}$ parameters are shown by the same symbols as in the top panel. The same parameters extracted from the LHF TDSE calculation are shown by orange triangles (derived from $A$ parameter) and blue asterisks ( $B$ parameter).

In Fig. 9 we compare $\beta_{2}$ and $\beta_{4}$ parameters as measured by Cirelli et al. [3] and those expressed in Eq. (11). On the top panel we compare $\beta_{2}$ and $\beta^{\mathrm{HH}}$ derived from the main harmonic peaks while on the bottom panel we display $\beta_{2}, \beta_{4}$ as measured directly from the SB amplitude and as expressed via $\beta^{\mathrm{SB}}(A)$ and $\beta^{\mathrm{SB}}(B)$ in Eq. (11). We see that the $\beta_{4}$ parameters compare rather favorably whereas the $\beta_{2}$ parameters are a bit higher than in the experiment.

The angular variations of the atomic time delay $\Delta \tau_{a}=$ $\tau_{a}\left(\theta_{k}\right)-\tau_{a}(0)$ in various sidebands of the Ar $3 p$ RABBITT trace and the Wigner time-delay angular variation $\Delta \tau_{\mathrm{W}}=$ $\tau_{\mathrm{W}}\left(\theta_{k}\right)-\tau_{\mathrm{W}}(0)$ at the same photon energies are displayed in Fig. 10 (top and bottom panels, respectively). In stark contrast to the analogous set of data for Ne $2 p$ shown in Fig. 4 , the angular variation of the Wigner time delay for Ar $3 p$ is of the same order of magnitude and is almost identical for SB30 near the CM. As a reference, in both panels of Fig. 10, the LOPT calculation [4] for SB32 is shown. Beyond the CM (SB48 and SB60), the angular variation of the Wigner time delay flattens whereas the same variation of the atomic time delay changes its sign and simultaneously lessens in magnitude.

In Fig. 11 we compare the angular variation of the atomic time delay $\Delta \tau_{a}=\tau_{a}\left(\theta_{k}\right)-\tau_{a}(0)$ in SB14 (top) and SB16. In the experiment [3], SB16 is tuned in resonance with the $4 s^{-1} 5 p$
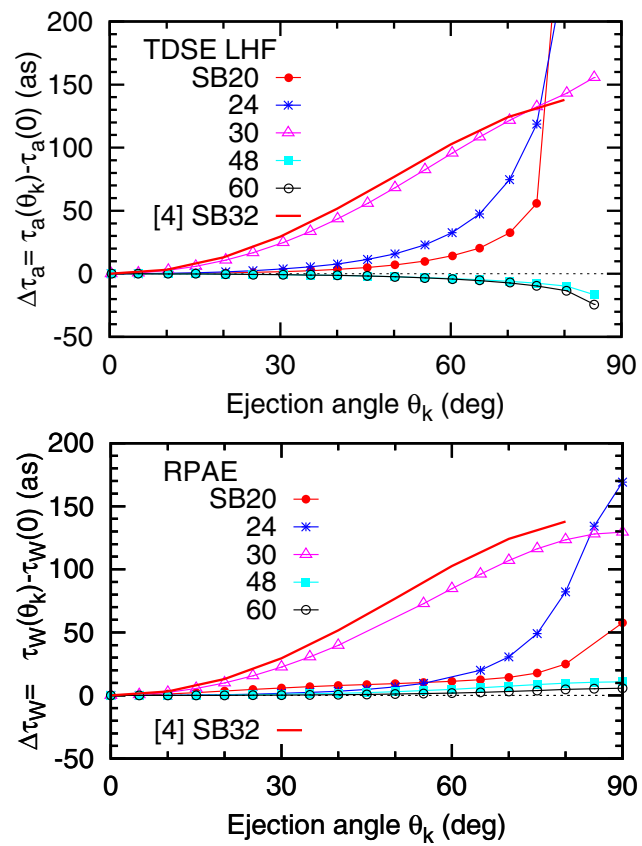

FIG. 10. (top) Angular variation of atomic time delay $\Delta \tau_{a}=$ $\tau_{a}\left(\theta_{k}\right)-\tau_{a}(0)$ in various sidebands of $\operatorname{Ar} 3 p$ RABBITT trace calculated with the LHF potential. (bottom) Angular variation of the Wigner time delay $\Delta \tau_{\mathrm{W}}=\tau_{\mathrm{W}}\left(\theta_{k}\right)-\tau_{\mathrm{W}}(0)$ from the XUV-only RPAE calculation. The angular variation of time delay for SB32 from Ref. [4] is shown for comparison.

autoionizing state while SB14 is off the resonance. For SB14 we find a fairly good agreement between the experiment and the present LHF TDSE calculation. The LOPT calculation
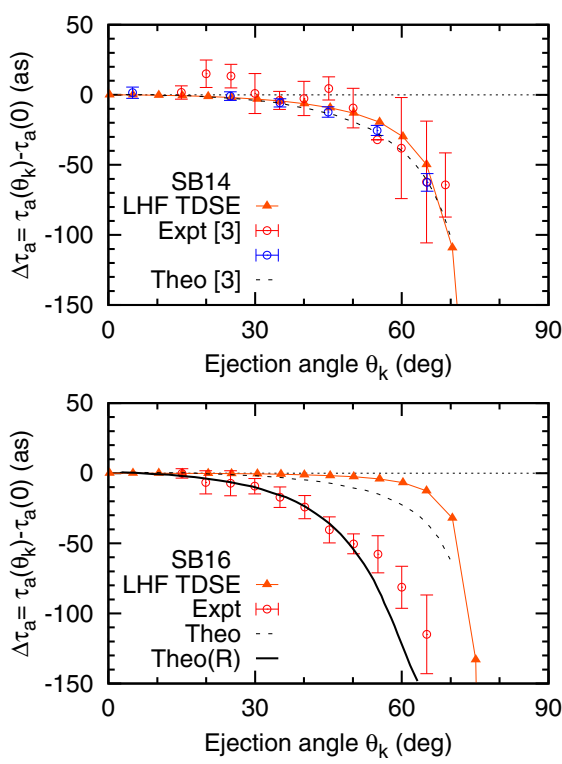

FIG. 11. Same as Fig. 10 for SB14 (top) and SB16 (bottom). Two sets of measurements from Ref. [3] are shown by open circles with error bars. The LOPT result from the same work is visualized by a dashed line. The LHF TDSE result is shown by orange triangles connected by the solid line. The bottom panel also shows the calculation from Ref. [3], which includes the Fano resonance (black solid line). 

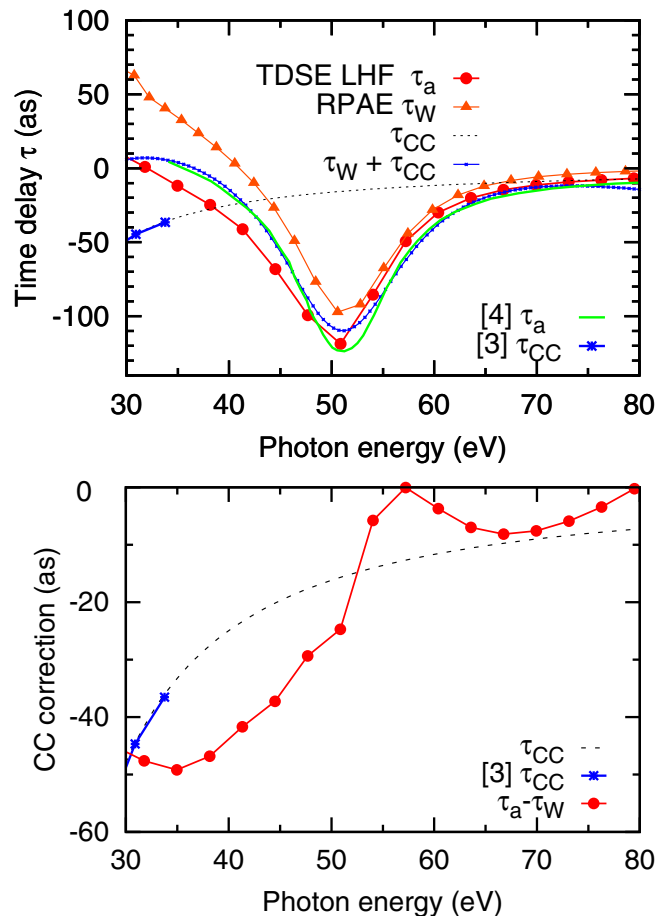

FIG. 12. Same as Fig. 5 for Ar $3 p$ shell. In addition, the atomic time delay $\tau_{a}$ from the LOPT calculation [4] and the CC correction $\tau_{\mathrm{CC}}=\left(\phi_{\mathrm{CC}}^{-}-\phi_{\mathrm{CC}}^{+}\right) / 2 \omega$ obtained from the phases $\phi_{\mathrm{CC}}^{ \pm}$reported in Ref. [3] are shown.

reported in Ref. [3] is also very close. For SB16 both the TDSE and LOPT calculations predict considerably weaker angular dependence than in the experiment and the calculation which accounts for resonance by the Fano configuration-interaction formalism.

Various time delays for the $\operatorname{Ar} 3 p$ shell in the zero-angle polarization direction are shown in Fig. 12. In the top panel, we display the atomic time delay $\tau_{a}$ from the TDSE LHF calculation, the Wigner time delay $\tau_{\mathrm{W}}$ from the RPAE calculation, the regularized hydrogenic $\mathrm{CC}$ correction $\tau_{\mathrm{CC}}$, and their sum $\tau_{\mathrm{W}}+$ $\tau_{\mathrm{CC}}$. We also show the atomic time delay $\tau_{a}$ from the LOPT calculation [4]. The latter is almost indistinguishable from the sum $\tau_{\mathrm{W}}+\tau_{\mathrm{CC}}$, but visibly different from the TDSE calculation for $\tau_{a}$. In the bottom panel we show the hydrogenic $\tau_{\mathrm{CC}}$ and the argon-specific value $\tau_{\mathrm{CC}}=\left(\phi_{\mathrm{CC}}^{-}-\phi_{\mathrm{CC}}^{+}\right) / 2 \omega$ obtained from the phases $\phi_{\mathrm{CC}}^{ \pm}$reported in Ref. [3]. Both values, which are remarkably close, are compared with the difference $\tau_{a}-\tau_{\mathrm{W}}$. Unlike in the Ne $2 p$ case, displayed in the bottom panel of Fig. 5, these two derivations of the CC correction give quite different results. This difference may, in principle, be attributed to the different approximations used in TDSE-LHF and RPAE calculations. The former employs a localized version of the HF potential and neglects the correlation while the latter gives the full account to the exchange and intershell correlation. However, the same calculations return quite similar sets of $\beta$ parameters. As such, it is more likely that the hydrogenic approximation to $\tau_{\mathrm{CC}}$ breaks for the argon $3 p$ shell.

This break down may have implications to theoretical interpretation of the time delay difference in the valence shell

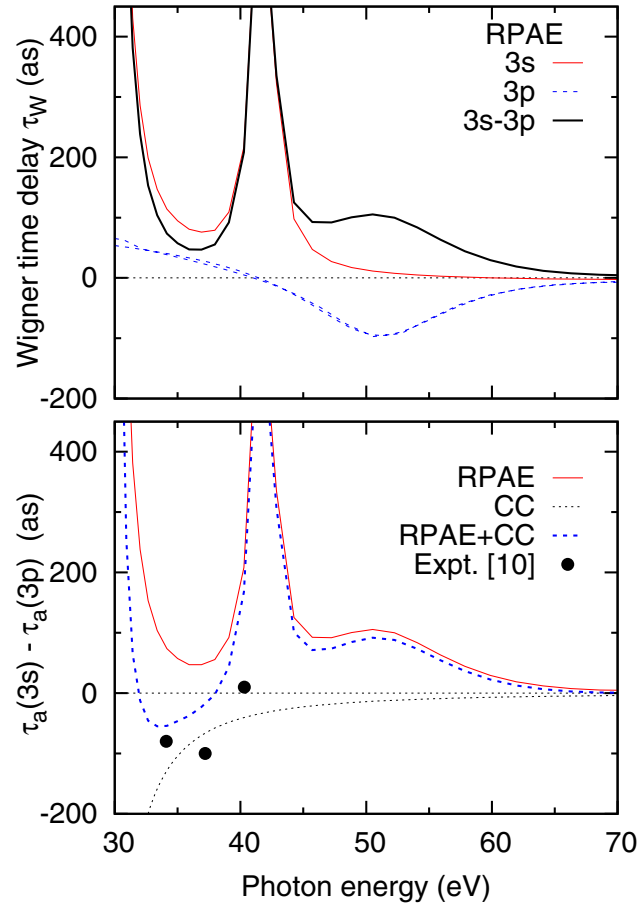

FIG. 13. (top) Wigner time delay in the $3 s$ and $3 p$ shells of $\mathrm{Ar}$ and their difference. (bottom) Atomic time-delay difference $\tau_{a}(3 s)-$ $\tau_{a}(3 p)$ as measured experimentally by Guénot et al. [10] (filled circles). The Wigner time-delay difference $\tau_{\mathrm{W}}(3 s)-\tau_{\mathrm{W}}(3 p)$ (red solid line) is augmented by the $\mathrm{CC}$ correction difference $\tau_{\mathrm{CC}}(3 s)-\tau_{\mathrm{CC}}(3 p)$ (dotted line) to get the atomic time-delay difference $\tau_{a}(3 s)-\tau_{a}(3 p)$ (blue dashed line).

of Ar shown in Fig. 13. Here the atomic time-delay difference

$$
\tau_{a}(3 s)-\tau_{a}(3 p)=\tau_{\mathrm{W}}(3 s)-\tau_{\mathrm{W}}(3 p)+\tau_{\mathrm{CC}}(3 s)-\tau_{\mathrm{CC}}(3 p)
$$

is computed with the hydrogenic CC corrections and compared with the RABBITT measurement [10]. As $\tau_{\mathrm{CC}}(3 p)$ deduced from the present TDSE calculation is more negative by about 20 as near the $40 \mathrm{eV}$ mark as compared with the hydrogenic estimate, the atomic time-delay difference estimated from Eq. (21) will be shifted upwards by the same amount. It will make the disagreement with the measurement [10] even worse. The present TDSE calculation is not able to give an estimate to $\tau_{\mathrm{CC}}(3 s)$ because ionization of this shell is strongly correlated with that of the valence $3 p$ shell and goes beyond the SAE approximation.

\section{Krypton $4 p$ shell}

We test validity of various effective potentials for $\mathrm{Kr}$ by determining the $\mathrm{CM}$ position in the $4 p$ photoionization cross section. We do so by comparing the squared radial integrals (20) calculated with the bound-state $4 p$ orbital and the continuous $d$ wave obtained from the radial Schrödinger equation (3). This comparison is shown in Fig. 14. Unlike in the case of Ar $3 p$ photoionization, illustrated in Fig. 8, the CM position calculated in the HF and RPAE differs by nearly $20 \mathrm{eV}$. This is so because of the influence of the intershell correlation between the $4 p$ and $3 d$ shells and which is accounted for in the 


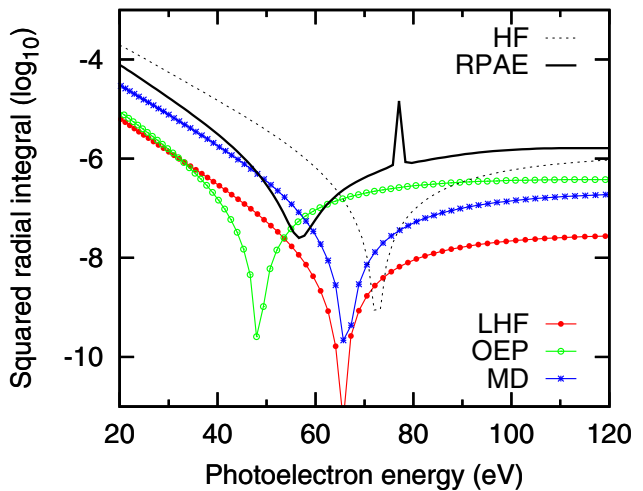

FIG. 14. The squared radial integral (20) calculated with the LHF (red filled circles), OEP (open green circles), Miller and Dow [24] (blue asterisks) potentials for Kr. The HF and RPAE results are shown with black dotted and solid lines, respectively.

RPAE but not in the HF calculation. This correlation is absent in the case of Ar $3 p$ as the $3 d$ shell is vacant for this atom. The CM position calculated with the LHF and MD potentials is in between the HF and RPAE whereas the OEP calculation displaces the $\mathrm{CM}$ to lower energies very significantly. We discard the OEP in the following.

The three sets of angular anisotropy $\beta$ parameters extracted from the high-harmonic peaks and the side bands are shown in Fig. 15 calculated with the LHF (top) and MD (bottom) potentials. We see that agreement between the TDSE and
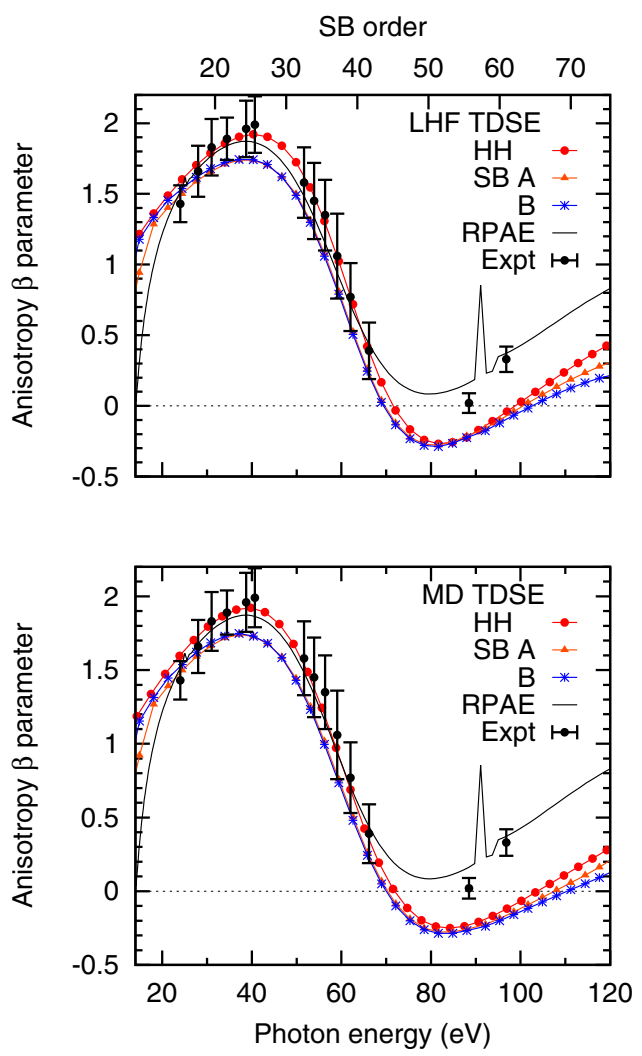

FIG. 15. Same as Fig. 3 for the $\mathrm{Kr} 4 p$ shell. The experimental data are from Ref. [33].

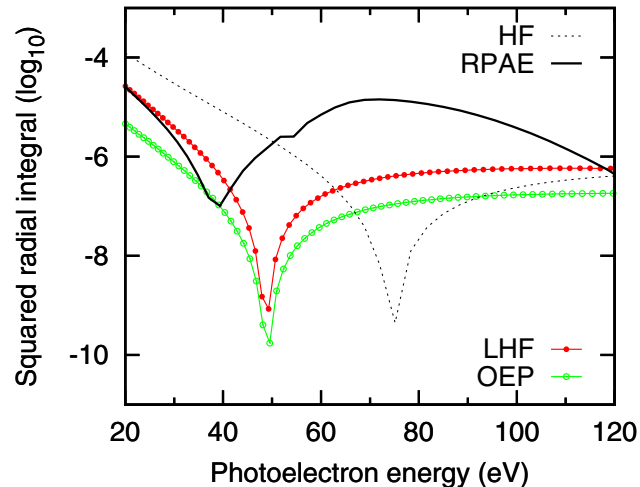

FIG. 16. The squared radial integral (20) calculated with the LHF (red filled circles), and OEP (open green circles) potentials for Xe. The HF and RPAE results are given by the black dotted and solid lines, respectively.

RPAE calculations is generally good but these calculations diverge at higher photon energies. This occurs well below the $3 d$ threshold whose position can be identified by the converging autoionization resonances visible in the RPAE curve. Experiment [33] clearly favors the RPAE calculation. Partial agreement between the TDSE calculations with the LHF and MD potentials, the RPAE and the experiment may be somewhat fortuitous given a strong deviation of the TDSE binding energies from the experimental threshold (see Table I). Should the $\beta$ parameters in Fig. 15 be plotted versus the photoelectron energy, this agreement will disappear.

\section{Xenon $5 p$ shell}

This tendency of deviation of the TDSE calculations with various local potentials from the RPAE and experiment is aggravated further in Xe. As an illustration, we show in Fig. 16 the $\mathrm{CM}$ position deduced from the squared radial integral (20). First, we observe that the HF and RPAE results diverge by as much as $40 \mathrm{eV}$. This is a clear sign of a very strong correlation between the $5 p$ and $4 d$ shells accounted for in the RPAE but missing in the HF. Second, both the LHF and OEP give the $\mathrm{CM}$ position which is displaced by $20 \mathrm{eV}$ from the RPAE for the same reason.

It is well known that missing the intershell correlation between the $5 p$ and $4 d$ shells in Xe has a profound effect on the anisotropy $\beta$ parameter. It becomes strongly displaced relative to the experiment as shown graphically in Fig. 1 of Ref. [34]. We therefore do not expect any reasonable agreement of the presently employed TDSE-SAE model with the experiment, either.

\section{CONCLUSIONS}

We presented a series of simulations and their analysis for the angular-dependent RABBITT traces in the valence shells of noble-gas atoms from $\mathrm{Ne}$ to Xe. Our simulations are based on numerical solutions of the one-electron TDSE driven with the XUV ionizing field and the IR probing pulse. Exchange between the departing photoelectron and the ionized atomic core is accounted for by various effective one-electron potentials. The accuracy of this account is tested by making 
comparison with the Hartree-Fock approximation which includes the exchange by constriction. The intershell correlation between the valence $n p$ and subvalent $n s,(n-1) d$ shells are neglected in a one-electron TDSE. To elucidate the strength of this correlation, we compare the TDSE results with the RPAE calculation which is known to account for the intershell correlation very accurately. However, the RPAE is unable to account for ultrafast electron dynamics and designed for much slower ionization processes initiated by long pulses of synchrotron radiation.

We focus our analysis on the anisotropy $\beta$ parameter, which is extracted from the angular dependence of the high-harmonic peaks as well as sideband RABBITT oscillation amplitude $A$ and $B$ factors. Within the scope of the soft photon approximation, all the three sets of $\beta$ should be in agreement which was found to be the case. This streamlines considerably the analysis of an angular-resolved RABBITT measurement and makes redundant the introduction of multiple sets of angular anisotropy parameters which was made by Cirelli et al. [3]. The phase of the RABBITT oscillation is converted to the angular-dependent time delay which is compared with the RPAE calculations. The time delay in the polarization direction is used to test accuracy of the hydrogenic CC correction.

Our results can be broadly categorized into the two groups. In lighter atoms, $\mathrm{Ne}$ and $\mathrm{Ar}$, the single-active-electron model is generally valid. The Ne calculations are particularly robust with all the tested effective potentials producing accurate results close to the RPAE predictions both for the angular anisotropy and the time delay. In Ar, because of the appearance of the Cooper minimum, the TDSE calculations become very sensitive to the choice of the effective potential and a simple analytic fit to the localized HF potential produces the best results for $\beta$ parameters. At the same time, this calculation suggests deviation of the CC correction from the regularized hydrogenic expression. Because of the Cooper minimum, the angular variation of the Wigner time delay is of the same magnitude as the variation of the atomic time delay. In $\mathrm{Ne}$, the angular variation of the Wigner time delay is negligible.

In heavier atoms, in $\mathrm{Kr}$ and particularly in $\mathrm{Xe}$, the intershell correlation between the valence $n p$ shell and subvalent $(n-$ 1) $d$ shell becomes very strong. In $\mathrm{Kr}$, with some choice of effective potentials, the present model can return sensible Cooper minimum position and $\beta$ parameters away from the $(n-1) d$ shell threshold. In $\mathrm{Xe}$, no effective potential is expected to replace the strong effect of intershell correlation and the present model is generally invalid.

Our findings are of importance to the theoretical analysis of angular-resolved RABBITT measurements. Particularly that there is a linear dependence of the $\beta_{2}$ and $\beta_{4}$ parameters which can be derived from the single set describing the whole RABBITT measurement, both the high-harmonic peaks and the side bands. This set can be easily compared with predictions of the RPAE theory which is valid for all noble-gas atoms. These $\beta$ parameters can also be tested against the XUV only measurements [28,30,32-34].

This work is a step forward in resolving the persistent controversy in the time-delay measurement in Ar [10]. However, as the measurement involved both the valence $3 p$ and the subvalent $3 s$ shells, we are unable to conclusively do so. The $3 s$ shell in argon is strongly correlated with the $3 p$ shell and this intershell correlation goes beyond the scope of the present model.

The model, as it stands now, can be applied to the subvalent $\mathrm{Kr} 3 d$ and $\mathrm{Xe} 4 d$ shells which are not effected strongly by intershell correlation with outer valence shells. The $n d$ correlation with the inner core is only noticeable near corresponding deeper thresholds. We can also easily incorporate the effect of a fullerene cage [35] to model a RABBITT process in encapsulated atoms. Eventually, we will attempt to generalize our model to account for intershell correlation. This will require considerable development of the existing one-electron TDSE code.

\section{ACKNOWLEDGMENTS}

We acknowledge our appreciation to Igor Ivanov for sharing his expertise in numerical modeling of RABBITT. The authors are also greatly indebted to Serguei Patchkovskii who placed his iSURF TDSE code at their disposal. Resources of the National Computational Infrastructure facility were employed.

\section{APPENDIX: RABBITT IN SOFT PHOTON APPROXIMATION}

We start from Eqs. (10) and (11) of Ref. [20] and write the amplitude of the XUV photon absorption modulated by absorption (+) or emission (-) of $n$ IR photons as

$$
\begin{aligned}
& S=\sum_{n=-\infty}^{+\infty} S_{n} \delta\left(k^{2} / 2-E_{0}-\omega_{x}-n \omega\right), \\
& S_{n}=-2 \pi i J_{-n}\left(\boldsymbol{a}_{0} \cdot \boldsymbol{k}_{n}\right) \exp \left[-i\left(\phi_{x}+n \phi\right)\right]\left\langle\boldsymbol{k}_{n}|\boldsymbol{\epsilon} \cdot \nabla| i\right\rangle,(\mathrm{A} 1)
\end{aligned}
$$

with $k_{n} \simeq\left[2\left(E_{0}+\omega_{x}+n \omega\right)\right]^{1 / 2}$ being the shifted momentum of the photoelectron and $\omega_{x}, \phi_{x}$ and $\omega, \phi$ as the XUV and IR frequencies and phase shifts, respectively. Here the matrix element $\left\langle\boldsymbol{k}_{n}|\boldsymbol{\epsilon} \cdot \nabla| i\right\rangle$ of the XUV photon absorption is written in the velocity gauge.

In RABBITT we are only interested in the $n=1$ and $n=-1$ sidebands. Their corresponding amplitudes are

$S_{ \pm 1} \equiv \mathcal{M}_{\boldsymbol{k}_{ \pm 1}}^{( \pm)}=-2 \pi i J_{\mp 1}\left(\boldsymbol{a}_{0} \cdot \boldsymbol{k}_{ \pm 1}\right) \exp \left[\mp i \phi_{\mathrm{CC}}^{ \pm}\right]\left\langle\boldsymbol{k}_{ \pm 1}|\boldsymbol{\epsilon} \cdot \nabla| i\right\rangle$.

Here we introduced the phase $\phi_{\mathrm{CC}}^{ \pm}$associated with the continuum-continuum transition in absorption or emission of an IR photon. For simplicity we have dropped the XUV phase $\phi_{x}$ and thus neglected the harmonic group delay. Using the transformation $J_{-n}=(-1)^{n} J_{n}$ we write

$$
\begin{aligned}
S_{+1}+S_{-1}= & -2 \pi i\left[J_{1}\left(\boldsymbol{a}_{0} \cdot \boldsymbol{k}_{-1}\right)\left\langle\boldsymbol{k}_{-1}|\boldsymbol{\epsilon} \cdot \nabla| i\right\rangle e^{-i \phi_{\mathrm{CC}}^{+}}\right. \\
& \left.-J_{1}\left(\boldsymbol{a}_{0} \cdot \boldsymbol{k}_{+1}\right)\left\langle\boldsymbol{k}_{+1}|\boldsymbol{\epsilon} \cdot \nabla| i\right\rangle e^{+i \phi_{\mathrm{CC}}^{-}}\right] .
\end{aligned}
$$

We can relate the phases of the dipole matrix elements with the soft photon shifted momenta by the phase energy derivative,

$$
\begin{aligned}
\left\langle\boldsymbol{k}_{ \pm 1}|\boldsymbol{\epsilon} \cdot \nabla| i\right\rangle & \approx\langle\boldsymbol{k}|\boldsymbol{\epsilon} \cdot \nabla| i\rangle e^{ \pm i \omega \partial \delta_{m_{i}}(\boldsymbol{k}) / \partial E} \\
\delta_{m_{i}}(\boldsymbol{k}) & =\arg \langle\boldsymbol{k}|\boldsymbol{\epsilon} \cdot \nabla| i\rangle
\end{aligned}
$$

Furthermore, we assume $J_{1}\left(\boldsymbol{\alpha}_{0} \cdot \boldsymbol{k}_{ \pm 1}\right) \approx J_{1}\left(\boldsymbol{\alpha}_{0} \cdot \boldsymbol{k}\right)$ and subsequently find the magnitude of the RABBITT signal (8) to be 
proportional to

$$
\begin{aligned}
\operatorname{Re}\left[\mathcal{M}_{\boldsymbol{k}}^{(-)} \mathcal{M}_{\boldsymbol{k}}^{*(+)}\right] & \propto\left|J_{1}\left(\boldsymbol{\alpha}_{0} \cdot \boldsymbol{k}\right)\right|^{2}\left[\sum_{m_{i}}\left|\left\langle\chi_{\boldsymbol{k}}|\boldsymbol{\epsilon} \cdot \nabla| \psi_{i}\right\rangle\right|^{2}\right] \\
& \propto \cos ^{2} \theta\left[1+\beta P_{2}(\cos \theta)\right]
\end{aligned}
$$

Here we used the expansion $J_{1}(x) \simeq x / 2+O\left(x^{3}\right)$ valid for a weak IR field and accordingly small parameter $\boldsymbol{\alpha}_{0}=\boldsymbol{F}_{0} / \omega^{2}$. We also performed the angular-momentum projection summation [36]

$$
\sum_{m_{i}}|\langle\boldsymbol{k}|\boldsymbol{\epsilon} \cdot \nabla| i\rangle|^{2} \propto \frac{\sigma_{i}}{4 \pi}\left[1+\beta P_{2}(\cos \theta)\right],
$$

where $\beta$ is the angular anisotropy parameter and $\sigma_{i}$ is the photoionization cross section of the $i$ th atomic shell.

The atomic time delay is given by

$$
\begin{aligned}
\tau & =\frac{1}{2 \omega} \arg \left[\mathcal{M}_{\boldsymbol{k}}^{(-)} \mathcal{M}_{\boldsymbol{k}}^{*(+)}\right] \\
& \equiv \frac{1}{2 \omega} \arg \left[\sum_{m_{i}}\left|c_{m_{i}}\right|^{2} e^{2 i \phi_{m_{i}}}\right]+\frac{\phi_{\mathrm{CC}}^{-}-\phi_{\mathrm{CC}}^{+}}{2 \omega} \\
& \equiv \tau_{\mathrm{W}}+\tau_{\mathrm{CC}},
\end{aligned}
$$

where we have used the shorthand

$$
\phi_{m_{i}}=\omega \frac{\partial \delta_{m_{i}}(\boldsymbol{k})}{\partial E}, \quad c_{m_{i}}=\left\langle\chi_{\boldsymbol{k}}|\boldsymbol{\epsilon} \cdot \nabla| \psi_{i}\right\rangle,
$$

for the quantities associated with the XUV photon absorption which define the Wigner time delay $\tau_{\mathrm{W}}$.
[1] G. Laurent, W. Cao, H. Li, Z. Wang, I. Ben-Itzhak, and C. L. Cocke, Attosecond Control of Orbital Parity Mix Interferences and the Relative Phase of Even and Odd Harmonics in an Attosecond Pulse Train, Phys. Rev. Lett. 109, 083001 (2012).

[2] S. Heuser, A. Jiménez Galán, C. Cirelli, C. Marante, M. Sabbar, R. Boge, M. Lucchini, L. Gallmann, I. Ivanov, A. S. Kheifets et al., Angular dependence of photoemission time delay in helium, Phys. Rev. A 94, 063409 (2016).

[3] C. Cirelli, C. Marante, S. Heuser, C. L. M. Petersson, A. J. Galán, L. Argenti, S. Zhong, D. Busto, M. Isinger, S. Nandi et al., Anisotropic photoemission time delays close to a Fano resonance, Nat. Commun. 9, 955 (2018).

[4] J. M. Dahlstrm and E. Lindroth, Study of attosecond delays using perturbation diagrams and exterior complex scaling, J. Phys. B: At. Mol. Opt. Phys. 47, 124012 (2014).

[5] P. Hockett, Angle-resolved RABBITT: Theory and numerics, J. Phys. B: At. Mol. Opt. Phys. 50, 154002 (2017).

[6] I. A. Ivanov and A. S. Kheifets, Angle-dependent time delay in two-color XUV + IR photoemission of He and Ne, Phys. Rev. A 96, 013408 (2017).

[7] H. Muller, Reconstruction of attosecond harmonic beating by interference of two-photon transitions, Appl. Phys. B: Lasers Opt. 74, s17 (2002).

[8] E. S. Toma and H. G. Muller, Calculation of matrix elements for mixed extreme-ultraviolet infrared two-photon above-threshold ionization of argon, J. Phys. B: At. Mol. Opt. Phys. 35, 3435 (2002).

[9] A. Sarsa, F. J. Gálvez, and E. Buendia, Parameterized optimized effective potential for the ground state of the atoms He through Xe, At. Data Nucl. Data Tables 88, 163 (2004).

[10] D. Guénot, K. Klünder, C. L. Arnold, D. Kroon, J. M. Dahlström, M. Miranda, T. Fordell, M. Gisselbrecht, P. Johnsson, J. Mauritsson et al., Photoemission-time-delay measurements and calculations close to the $3 \mathrm{~s}$-ionization-cross-section minimum in Ar, Phys. Rev. A 85, 053424 (2012).

[11] M. Isinger, R. Squibb, D. Busto, S. Zhong, A. Harth, D. Kroon, S. Nandi, C. L. Arnold, M. Miranda, J. M. Dahlström et al., Photoionization in the time and frequency domain, Science 358, 893 (2017).
[12] M. Schultze et al., Delay in photoemission, Science 328, 1658 (2010).

[13] A. S. Kheifets and I. A. Ivanov, Delay in Atomic Photoionization, Phys. Rev. Lett. 105, 233002 (2010).

[14] L. R. Moore, M. A. Lysaght, J. S. Parker, H. W. van der Hart, and K. T. Taylor, Time delay between photoemission from the $2 p$ and $2 s$ subshells of neon, Phys. Rev. A 84, 061404 (2011).

[15] J. M. Dahlström, T. Carette, and E. Lindroth, Diagrammatic approach to attosecond delays in photoionization, Phys. Rev. A 86, 061402 (2012).

[16] A. S. Kheifets, Time delay in valence-shell photoionization of noble-gas atoms, Phys. Rev. A 87, 063404 (2013).

[17] J. Feist, O. Zatsarinny, S. Nagele, R. Pazourek, J. Burgdörfer, X. Guan, K. Bartschat, and B. I. Schneider, Time delays for attosecond streaking in photoionization of neon, Phys. Rev. A 89, 033417 (2014).

[18] J. J. Omiste and L. B. Madsen, Attosecond photoionization dynamics in neon, Phys. Rev. A 97, 013422 (2018).

[19] F. Morales, T. Bredtmann, and S. Patchkovskii, iSURF: A family of infinite-time surface flux methods, J. Phys. B: At. Mol. Opt. Phys. 49, 245001 (2016).

[20] A. Maquet and R. Taïeb, Two-colour IR + XUV spectroscopies: The "soft-photon approximation,” J. Mod. Opt. 54, 1847 (2007).

[21] A. S. Kheifets, A. Mandal, P. C. Deshmukh, V. K. Dolmatov, D. A. Keating, and S. T. Manson, Relativistic calculations of angle-dependent photoemission time delay, Phys. Rev. A 94, 013423 (2016).

[22] J. Dahlström, D. Guénot, K. Klünder, M. Gisselbrecht, J. Mauritsson, A. L. Huillier, A. Maquet, and R. Taïeb, Theory of attosecond delays in laser-assisted photoionization, Chem. Phys. 414, 53 (2012).

[23] J. C. Slater, A simplification of the Hartree-Fock method, Phys. Rev. 81, 385 (1951).

[24] D. L. Miller and J. D. Dow, Atomic pseudopotentials for soft X-ray excitations, Phys. Lett. A 60, 16 (1977).

[25] R. O. Jones and O. Gunnarsson, The density functional formalism, its applications and prospects, Rev. Mod. Phys. 61, 689 (1989). 
[26] G. Wendin and A. F. Starace, Perturbation theory in a stronginteraction regime with application to $4 d$-subshell spectra of $\mathrm{Ba}$ and La, J. Phys. B: At. Mol. Phys. 11, 4119 (1978).

[27] Y. Ralchenko, A. E. Kramida, J. Reader, and NIST ASD Team, NIST atomic spectra database, version 3.1.5 (2011), http://physics.nist.gov/asd

[28] K. Codling, R. G. Houlgate, J. B. West, and P. R. Woodruff, Angular distribution and photoionization measurements on the $2 p$ and $2 s$ electrons in neon, J. Phys. B: At. Mol. Phys. 9, L83 (1976).

[29] U. Fano, Propensity rules: An analytical approach, Phys. Rev. A 32, 617 (1985)

[30] R. G. Houlgate, K. Codling, G. V. Marr, and J. B. West, Angular distribution and photoionization cross section measurements on the $3 p$ and $3 s$ subshells of argon, J. Phys. B: At. Mol. Phys. 7, L470 (1974).

[31] J. Mauritsson, M. B. Gaarde, and K. J. Schafer, Accessing properties of electron wave packets generated by attosecond pulse trains through time-dependent calculations, Phys. Rev. A 72, 013401 (2005).
[32] S. Southworth, A. Parr, J. Hardis, J. Dehmer, and D. Holland, Calibration of a monochromator/spectrometer system for the measurement of photoelectron angular distributions and branching ratios, Nucl. Instrum. Methods Phys. Res. Sect. A 246, 782 (1986).

[33] D. L. Miller, J. D. Dow, R. G. Houlgate, G. V. Marr, and J. B. West, The photoionisation of krypton atoms: a comparison of pseudopotential calculations with experimental data for the $4 p$ asymmetry parameter and cross section as a function of the energy of the ejected photoelectrons, J. Phys. B: At. Mol. Phys. 10, 3205 (1977).

[34] J. B. West, Progress in photoionization spectroscopy of atoms and molecules: An experimental viewpoint, Appl. Opt. 19, 4063 (1980).

[35] A. Mandal, P. C. Deshmukh, A. S. Kheifets, V. K. Dolmatov, and S. T. Manson, Angle-resolved Wigner time delay in atomic photoionization: The $4 d$ subshell of free and confined Xe, Phys. Rev. A 96, 053407 (2017).

[36] M. Y. Amusia, Atomic Photoeffect (Plenum Press, New York, 1990). 